\title{
Tonsil fibromatosis
}

\section{Juliana Sato ${ }^{1}$, Antonio Augusto de Lima Pontes ${ }^{2}$, Ricardo Frazatto ${ }^{3}$, Reginaldo Raimundo Fujita ${ }^{4}$}

Keywords: tonsil, fibromatosis.

\section{INTRODUCTION}

Fibromatosis is a non-metastatic, however locally invasive tumor that may infiltrate muscle, fat, and bone. It may be fatal depending on its location. Tumors in the head and neck are particularly dangerous ${ }^{1}$

These fibroblast tumors have poorly defined margins and are not encapsulated $^{2}$. Anaplasia and invasion of nerves and vessels are not present; mitosis is rare and there may be collagen between tumor cells ${ }^{1}$.

This paper aims to present a case of fibromatosis involving a child's tonsils and discuss issues related to treatment and prognosis.

\section{CASE REPORT}

The patient is a 10-year-old male, brown, born in Maceió. In 2002, he came to our service with a history of snoring, mouth breathing, and repetition tonsillitis. He underwent an adenoidectomy at the age of four. In the examination he was seen to

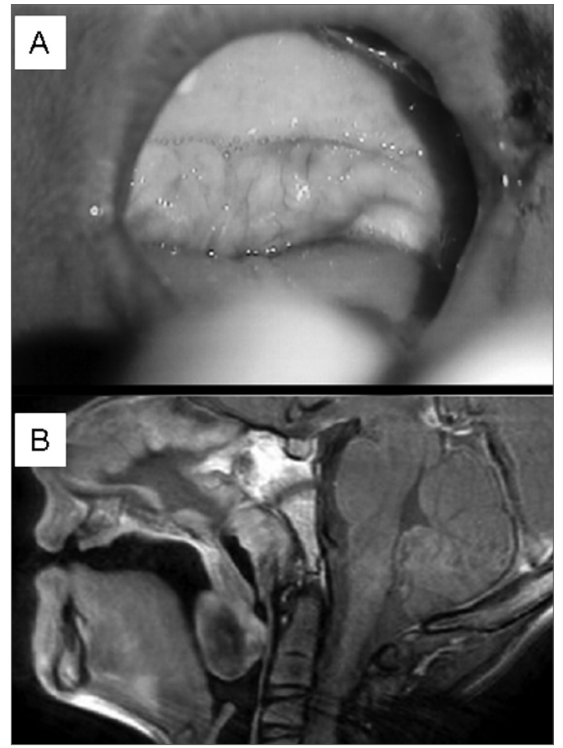

Figure 1. Tumor invading the soft palate and tonsillar pillars. have grade IV tonsil hypertrophy and reduced air column in the rhinopharynx as seen in the radiography of the cavum, being thus referred to an adenotonsillectomy.

During surgery it was verified that his tonsils were unusually hard, and adhesions between the tongue base and the uvula were observed. The adenotonsillectomy was performed and tonsil pathology tests were positive for a fibroblastic proliferation consistent with fibromatosis.

A year later the patient began to lose weight and experience difficulty swallowing solid foods. Oroscopy revealed a tumor invading the soft palate and tonsillar pillars (Figure). Laryngoscopy did not show any involvement of the pharyngeal posterior wall. The MRI revealed a solid, well-limited lesion involving distal portions of the soft palate, measuring $3.0 \times 2.5 \times 2.5 \mathrm{~cm}$ with marginal enhancement after contrast uptake. Hard palate, tongue base, posterior pharyngeal wall, and skull base were not involved. He was referred to surgical resection of the lesion with a safety margin of $2.0 \mathrm{~cm}$.

\section{DISCUSSION}

Fibromatosis occurs in the head and neck in $10-35 \%$ of the cases 1 . Stout was the first to identify and describe the disease's aggressive manifestation in $1954^{3}$. This disease affects predominantly children and young adults, and develops slowly in about half the patients ${ }^{2}$.

Resection with broad safety margins is the treatment of choice, a challenging goal for head and neck tumor removal without leaving any sequelae $e^{1,2,4}$. In adults, the recommended safety margin is three centimeters ${ }^{4}$. Post-surgery relapse is seen in $23.8 \%$ to $57 \%$ of the patients ${ }^{5}$. Some authors indicate radical clearance for patients with neck fibromatosis ${ }^{1}$.

Fowler ${ }^{5}$ studied 31 cases of fibromatosis in the oral cavity, and found that the cheeks, tongue, and submaxillary region were the most commonly affected sites.

There is no consensus as to the use of chemo or radiotherapy, but both have been used as adjuvant treatment in inoperable tumors. One of the downsides of radiotherapy is the high dosage required to treat the tumor, as it may damage bone epiphysis and thus impair the patient's growth ${ }^{2,4}$.

Chemotherapy alone does not seem to be a curative treatment, but may be effective in managing inoperable tumors and in reducing the size of the tumor before surgery. Some studies indicate that tumor growth may be impacted by estrogen, and that adjuvant therapy with tamoxifen might be useful. Others looked into non-steroidal anti-inflammatory drugs due to their impact in prostaglandin metabolization ${ }^{4}$.

In the literature we found only one case of tonsil fibromatosis, also in a child, with invasion of the pterygopalatine fossa Cure was only possible through broad tumor resection ${ }^{6}$

\section{CONCLUSION}

This paper illustrates the difficulties in treating a rare condition as head and neck fibromatosis in children. Despite the various treatments proposed for the disease, not all can be offered to children, thus turning it into a benign disease of challenging management.

\section{REFERENCES}

1.Masson JK, Soule EH. Desmoid tumors of the head and neck. Am J Surg 1966;112(4):61522.

2.Hill DR, Newman H, Phillips TL. Radiation therapy of desmoid tumors. Am J Roentgenol Radium Ther Nucl Med 1973;117(1):84-9.

3.Stout AP. Juvenile fibromatoses.Cancer 1954;7(5):953-6.

4.Plukker JT, van Oort I, VermeyA, Molenaar I, Hoekstra HJ, Panders AK et al. Aggressive fibromatosis: therapeutic problems and the role of adjuvant radiotherapy. Br J Surg 1995;82(4):510-4.

5.Fowler CB, Hartman KS, Brannon RB. Fibromatosis of the oral and paraoral region. Oral Surg Oral Med Oral Pathol 1994;77(4):37385.

6.Tostevin PMJ, Wyatt M, Hosni A. Sixcases of fibromatosis of the head and neck in children. Int J Pediatr Otorhinolaryngol 2000;53(3):235-44.

ENT, Fellow in pediatric otorhinolaryngology at UNIFESP- EPM MD, third-year specialization student at Unifesp-EPM.

MSc in otorhinolaryngology and head and neck surgery at UNIFESP-EPM, ENT.

Adjunct Professor at the Department of Otorhinolaryngology and Head and Neck Surgery at UNIFESP-EPM, Head of the Pediatric ENT Clinic at UNIFESP-EPM. Universidade Federal de São Paulo - Escola Paulista de Medicina

Send correspondence to: Juliana Sato - Rua Leandro Dupret 662 apto 133 Vila Clementino São Paulo SP 04025-013.

Paper submitted to the RBORL-SGP (Publishing Management System - Brazilian Journal of Otorhinolaryngology) on September 3, 2005 and approved on June 28, 2007. This paper was submitted to the RBORL-SGP (Publishing Manager System) on 3 September 2005. code 1042.

The article was accepted on 28 June 2007 . 